УДК 336.763.21, 330.43

JEL G11, C01, C55, C58

DOI 10.25205/2542-0429-2020-20-3-48-69

\title{
Performance of Estimators of Quadratic Variation Based on High Frequency Data Empirical Review
}

\author{
J. Gayomey ${ }^{1}$, A. V. Kostin ${ }^{1,2}$ \\ ${ }^{1}$ Novosibirsk State University \\ Novosibirsk, Russian Federation \\ ${ }^{2}$ Institute of Economics and Industrial Engineering SB RAS \\ Novosibirsk, Russian Federation
}

\begin{abstract}
Recently, advances in computer technology and data recording and storage have made high-frequency financial data readily available to researchers. As a result, the volatility literature has steadily progressed toward the use of higher-frequency data. However, the move towards the use of higher-frequency financial data in the estimation of volatility of financial returns has resulted in the development of many realised volatility measures of asset return variability based on a variety of different assumptions and functional forms and thus making theoretical comparison and selection of the estimators for empirical applications very difficult if not impossible. This article provides an empirical review on the performance of estimators of quadratic variation/integrated variance based on high-frequency data to aid their application in empirical analysis. The result of the review shows that no single estimator works best in all situations; however, the more sophisticated realised measures, in particular the TSRV and KRV, are superior to the other estimators in terms of their estimation accuracy in the presence of market microstructure noise.
\end{abstract}

Keywords

quadratic variation, integrated variance, realized variance, realised volatitlty, two scale realized variance, kernel realised variance, quantile realized variance, range realized variance, duration realized variance, bipower realized variance, jump robust estimators

For citation

Gayomey J., Kostin A. V. Performance of Estimators of Quadratic Variation Based on High Frequency Data. Empirical Review. World of Economics and Management, 2020, vol. 20, no. 3, p. 48-69. DOI 10.25205/ 2542-0429-2020-20-3-48-69

(C) Дж. Гайомей, А. В. Костин, 2020

ISSN 2542-0429

Мир экономики и управления. 2020. Том 20, № 3

World of Economics and Management, 2020, vol. 20, no. 3 


\title{
Оценщики квадратичной вариации, основанные на высокочастотных данных Эмпирический обзор
}

\author{
Дж. Гайомей ${ }^{1}$, А. В. Костин ${ }^{1,2}$ \\ ${ }^{1}$ Новосибирский национальный исследовательский государственный университет \\ Новосибирск, Россия \\ ${ }^{2}$ Институт экономики и организации промышленного производства СО РАН \\ Новосибирск, Россия
}

Аннотация

В последнее время достижения в области компьютерных технологий, записи и хранения данных позволили сделать финансовые данные доступными для исследователей. В результате литература о волатильности стала неуклонно развиваться в сторону использования чаще предоставляемых финансовых данных. Однако переход к использованию финансовых данных с более высокой степенью периодичности при оценке волатильности финансовой доходности привел к разработке многих реализованных показателей волатильности изменчивости доходности активов, основанных на множестве различных допущений и функциональных форм, тем самым крайне затрудняя проведение теоретических сравнений и выбор оценок для эмпирических приложений. В этой статье представлен эмпирический обзор эффективности оценок квадратичной вариации / интегрированной дисперсии на основе высокочастотных данных для упрощения их применения в эмпирическом анализе. В обзоре показано, что нельзя выделить ни одного их рассмотренных оценщиков, который работал бы лучше остальных во всех ситуациях, однако более сложные расчеты оценки волатильности, в частности на основе TSRV и KRV, превосходят другие аналоги с точки зрения точности оценки в присутствии рыночного микроструктурного шума.

Ключевые слова

квадратичная вариация, интегрированная дисперсия, реализованная дисперсия, реализованная волатильность, реализованная дисперсия по двум шкалам, реализованная дисперсия ядра, реализованная квантильная дисперсия, реализованная дисперсия диапазона, реализованная дисперсия продолжительности, реализованная двухстепенная дисперсия, робастные оценки скачка

Для циитирования

Гайомей Дж., Костин А. В. Оценщики квадратичной вариации, основанные на высокочастотных данных. Эмпирический обзор // Мир экономики и управления. 2020. Т. 20, № 3. С. 48-69. (на англ. яз.) DOI 10.25205/2542-0429-2020-20-3-48-69

\section{Introduction}

"Volatility is central to many applied issues in finance and financial engineering, ranging from asset pricing and asset allocation to risk management" [1, p. 106]. Proper appreciation of volatility and its dynamics is central to asset pricing. As the main measure of risk in finance, volatility is important in the determination of optimum portfolios, the pricing and hedging of derivative, the determination of a firm's exposure to various risk factors and the compensation it can expect to earn from those risk exposures. Volatility is also important in the search for trading and investment opportunities which guarantee an attractive risk-return trade-off. "Traditionally, researchers who wanted to extract and forecast financial volatility had to rely on data recorded at only moderate intervals: daily, for instance, or even monthly. But recently, data at much more frequent intervals - high-frequency data - have become increasingly available." [3, p. 294]. High-frequency financial data usually refers to observation sampled at a time horizon 
smaller than a trading day. Improvement in computer technology and data recording and storage have made these data sets readily available to researchers. Consequently, the volatility literature has steadily progressed towards the use of higher-frequency data. Thus, an exciting new area of research now involves estimating, modelling and forecasting conditional volatility and correlation using high frequency intra-day data. The main advantage of using high-frequency financial data to estimate volatility is in the increased quality of volatility forecast. High frequency data has been demonstrated to improve our ability to understand and forecast financial volatility.

However, the move towards the use of higher-frequency financial data in the estimation of volatility of financial returns has resulted in the development of numerous realized volatility estimators. These estimators are based on a variety of different assumptions about the price process and take many different functional forms. Additionally, the new estimators are based on different sampling schemes and price series, for example, one may be based upon sampling in calendar time with trade price while another utilizes tick time sampling with quote price. Also, some of the realized volatility estimators further require choices about tuning parameters such as a kernel bandwidth or ' block size' for their practical application. These, as well as other tractability issues often prohibit the theoretical asymptotic comparison of the various estimators. Since it is difficult to theoretically compare the different estimators, we must rely on empirical performance of the estimators to determine the most appropriate estimator for any given asset or empirical application.

In view of the above, the main goal of this article is to provide an empirical review on the performance of estimators of quadratic variation/integrated variance based on high-frequency data. In other to achieve the above goal, the following specific objectives were established:

1) to discuss the most popular and the most recent approaches to estimate quadratic variation/ integrated variance based on various characteristics of high-frequency financial data;

2) to summarize the asymptotic properties of the realized volatility estimators;

3) to provide an empirical evaluation of the performance of the realized volatility estimators based on the result of recent studies.

This review is particularly important for practitioners and researchers in the areas of asset pricing, asset allocation and risk management as far as their empirical analysis are concerned. The review in this article differs from existing ones in that, in addition to the theoretical review, it also provides an empirical evaluation of the estimation accuracy of the realized measures.

The rest of the paper is organised as follows. Section 2 presents a theoretical review of the most popular and the most recent estimators of quadratic variation together with their large sample properties. An empirical evaluation of the estimators based on the result of recent studies is presented in Section 3. In section 4, we draw conclusion.

\section{Review of Estimators of Quadratic Variation/Integrated Variance 2.1 Realized Volatility (RV)}

Let $S_{t}$ represent the price process of a financial asset. Let us also assume that the process $X_{t}=\log S_{t}$ is governed by an Itô process 


$$
d X_{t}=\mu_{t} d t+\sigma_{t} B_{t}
$$

where $\mu_{t}$ is the drift coefficient, $\sigma_{t}$ denotes the instantaneous volatility of $X_{t}$ (the returns process) and $B_{t}$ is a standard Brownian motion. Our aim is to estimate the volatility of the log price process given above. When $\sigma_{t}$ is assumed to be a (continuous) stochastic process, the object of interest is the quadratic variation $(\mathrm{QV})$ or integrated variance (IV),

$$
\langle X, X\rangle_{T}=\int_{0}^{T} \sigma^{2}(t) d t
$$

over a fixed time interval $[0, \mathrm{~T}]$. According to the volatility literature (see $[4,5,6]$ etc, for example), a natural estimator of $\langle X, X\rangle_{T}$, for example, over a single time interval $[0, \mathrm{~T}]$ is realized volatility or realized variance $(\mathrm{RV})$ which is defined as the sum of all the squared intraday log returns in $[0, \mathrm{~T}]$

$$
[X, X]_{T}=\sum_{i=1}^{n}\left(X_{t_{i+1}}-X_{t_{i}}\right)^{2}
$$

The above estimator is justified by the theoretical results in stochastic processes stating that

$$
\operatorname{plim} \sum_{i=1}^{n}\left(X_{t_{i+1}}-X_{t_{i}}\right)^{2}=\int_{0}^{T} \sigma_{t}^{2} d t
$$

as the sampling frequency increases. Thus, according to the above theory, realized volatility constructed from the highest-frequency data should give the best possible estimate for integrated volatility.

Andersen [5] showed that the realized variance computed using all data available is a consistent estimator of IV in the absence of market microstructure noise such that $R V_{t}^{(\text {all })} \stackrel{p}{\rightarrow} I V_{t}$. Barndor-Nielsen and Shephard [6] showed the consistency of the RV estimator and derived its asymptotic distribution as:

$$
\sqrt{n_{t}} \frac{1}{\sqrt{2 I Q_{t}}}\left(R V_{t}^{\text {all }}-I V_{t}\right) \stackrel{d}{\rightarrow} N(0,1)
$$

with

$$
\mathrm{IQ}=\int_{0}^{1} \sigma^{4}(t+\tau-1) d \tau
$$

denoting the integrated quarticity (IQ). To facilitate the application of the above asymptotic result, Barndor_Nielsen and Shephard [7] introduced the concept of realized power variation that allows us to estimate IQ via the realized quarticity (RQ):

$$
Q R^{\text {all }}=\frac{n_{t}}{3} \sum_{i=0}^{n_{t}} r_{t, i}^{4}
$$


such that

$$
\sqrt{n_{t}} \frac{1}{\sqrt{\frac{2}{3} R Q_{t}^{a l l}}}\left(R V^{(a l l)}-I V_{t}\right) \stackrel{d}{\rightarrow} \mathrm{N}(0,1)
$$

can be used for large $n$.

\subsection{Two Scales Realized Volatility (TSRV) Estimator}

Although realized volatility consistently estimate the price variation accumulated over some time interval, its consistency hinges on increasingly finer sampled highfrequency returns. In practice, the sampling frequency is limited by the actual quotation or transaction frequency. Moreover the very high frequency prices are contaminated by market microstructure effects such as bid-ask bounce effects, price discreteness etc., leading to biases in realized volatility (Pigorsch, Pigorsch, Popov [7], Andersen et al. [4]; Barndorff-Nielsen and Shephard [8]).

According to the high frequency volatility literature (see for example [2], [7], [9] [10]), when the observed $\log$ price at time $\mathrm{t}, Y_{t}$, is contaminated by market microstructure noise or measurement error, $Y_{t}$ can be taught of as comprising of a latent efficient / true price, $X_{t}$, and a microstructure noise, $\varepsilon_{t}$, that is:

$$
Y_{t}=X_{t}+\varepsilon_{t}
$$

As before, our interest is to estimate the quadratic variation, $\langle X, X\rangle_{T}=\int_{0}^{T} \sigma^{2}(t) d t$ over a fixed time period $[0, \mathrm{~T}]$. A natural estimator of $\langle X, X\rangle_{T}$ is realized volatility

$$
[Y, Y]_{T}=\sum_{i=1}^{n}\left(Y_{t_{i+1}}-Y_{t_{i}}\right)^{2}
$$

In the absence of microstructure noise, $[Y, Y]_{T}$ is a consistent estimator of $\langle X, X\rangle_{T}$. However, $Y_{t}$ is contaminated by market microstructure noise and ignoring the noise will lead to serious problems. With microstructure noise, after suitable scaling, RV constructed from the observed log-returns is a consistent and asymptotically normal estimator but of the quantity $2 n E[\varepsilon]^{2}$ instead of $\langle X, X\rangle_{T}$, the object of interest.

Zhang, Mykland and Ait-Sahalia [9] introduced the Two Scales Realized Volatility (TSRV) to consistently estimate $\langle X, X\rangle_{T}$ (quadratic variation) in the presence of market microstructure noise. The construction of the TSRV estimator is based on subsampling, averaging and bias-correction. The estimator computes a "subsampled RV on one or more slower time scales and then combine with RV constructed on a faster time scale to correct bias due to microstructure noise." [11, p. 3]. Aït-Sahalia and Yu [2] identified the following steps in the construction of the TSRV estimator:

1) First we partition the original grid of observation times, $G=\left\{t_{0}, \ldots, t_{n}\right\}$ into subsamples, $G^{k}, k=1, \ldots \ldots . K$, where $\mathrm{n} / \mathrm{K} \rightarrow \infty$ as $\mathrm{n} \rightarrow \infty$. For example, for $G^{(1)}$ start at the first observation and take an observation every 5 minutes; for $G^{(2)}$, start at the second observation and take an observation every 5 minutes, etc. 
2) Next, we average the RVs computed for each subsample. So far as there is a benefit to subsampling, this benefit can be maintained while the variation in the estimator is reduced through averaging. Averaging over the subsamples the estimators, $[Y, Y]_{T}^{k}$, results in the average realised volatility estimator:

$$
[Y, Y]_{T}^{a v g}=\frac{1}{K} \sum_{k=1}^{K}[Y, Y]_{T}^{k}
$$

3) Finally, we correct bias using realized volatility constructed from all the available intraday returns, that is $[Y, Y]_{T}^{\text {all }}$. The average realized volatility estimator is biased so a bias correction is carried out using realized volatility constructed from all observations.

Following the above steps, the TSRV can be expressed in the following form:

$$
\widehat{\langle\mathrm{X}, \mathrm{X}}\rangle_{\mathrm{T}}^{\text {tsrv }}=\underbrace{[\mathrm{Y}, \mathrm{Y}]_{\mathrm{T}}^{\text {avg }}}_{\text {slow time scale }}-\frac{\overline{\mathrm{n}}}{\mathrm{n}} \underbrace{[\mathrm{Y}, \mathrm{Y}]_{\mathrm{T}}^{\text {all }}}_{\text {fast time scale }}
$$

The TSRV estimator utilizes all the return data available in its estimation but still gives consistent estimates of both $\langle X, X\rangle_{T}$ and $E[\varepsilon]^{2}$. The estimator is a consistent and asymptotically unbiased estimator of quadratic variation under the independent noise assumption. It has the rate of convergence $n^{-1 / 6}$. Given an optimal number of subsamples $K^{*}$, determined as , $K^{*}=\mathrm{cm}^{2 / 3}$, the asymptotic distribution of the estimator under IID noise structure assumption is given by

$$
\widehat{\langle\mathrm{X}, \mathrm{X}}\rangle_{\mathrm{T}}^{\mathrm{tsrv}} \underset{\approx}{\mathcal{L}} \underbrace{[X, X]_{T}}_{\text {object of interest }}+\frac{1}{n^{1 / 6}} \underbrace{[\underbrace{\frac{8}{c^{2}} E\left[\varepsilon^{2}\right]^{2}}_{\text {due to noise }}+\underbrace{c \frac{4 T}{3}}_{\text {due to discretization }} \int_{0}^{T} \sigma_{t}^{4} d t}_{\text {total variance }} Z_{\text {total }}^{1 / 2}
$$

where

$$
c=\left\{\frac{1}{12 E\left[\left(\varepsilon_{t, i}^{2}\right)\right]^{2}}+I Q_{t}\right\}^{-1 / 3}
$$

in the case of equidistant observations. For small samples, a refinement to $\widehat{X, X_{T}}$ can be obtained as

$$
\widehat{\mathrm{X}, \mathrm{X}}_{\mathrm{T}}^{\mathrm{tsrv}, \operatorname{adj}}=\left(1-\frac{\bar{n}}{n}\right)^{-1} \widehat{\mathrm{X}, \mathrm{X}}_{\mathrm{T}}^{\mathrm{tsrv}}
$$

The estimators above are obtained under IID noise structure assumption. In order to account for possibly dependent noise, Ait-Sahalia, Mykland and Zhang [12] and Zhang [13] introduced a generalized version of the TSRV estimator also based on the two time scales idea. In order to obtain the generalized TSRV estimator, we first have to define the average lag $\mathrm{J} R \mathrm{R}, \mathrm{RV}_{\mathrm{t}, \mathrm{J}}^{(\mathrm{AL})}$, as shown below. 


$$
R V_{t, J}^{(A L)}=\frac{1}{J} \sum_{i=0}^{n_{t}-J}\left(r_{t, i+J}-r_{t, i}\right)^{2}
$$

Next, we construct the generalized TSRV estimator as follows

$$
R V_{t}^{(A M Z)}=R V_{t, K}^{A L}-\frac{\bar{n}_{t}^{(K)}}{\bar{n}_{t}^{(J)}} R V_{t, J}^{(A L)}
$$

with $\bar{n}_{t}^{(K)}=\frac{n_{t}-K+1}{K}, \bar{n}_{t}^{(J)}=\frac{n_{t}-J+1}{J}, 1 \leq J<K<n_{t}$. The adjusted estimator for small sample is given by

$$
R V_{t}^{(A M Z, a d j)}=\left(1-\frac{\bar{n}_{t}^{(K)}}{\bar{n}_{t}^{(J)}}\right)^{-1} R V_{t}^{(A M Z)}
$$

In order to account for serial correlation in the noise, the RVs in the generalized TSRV estimator above are based on overlapping $\boldsymbol{J}$-period intraday returns (Pigorsch [7]). The generalized TSRV estimator is consistent and asymptotically unbiased under time-dependent noise. However, it has a convergence rate of $n^{-1 / 6}$ which is below the optimal convergence rate of $n^{-1 / 4}$ in the fully parametric case. This led to the introduction of the multiple time scale estimator (MSRV) by Ait-Sahalia [2]. The multiple time scale estimator is based on the weighted average of average lag- $\boldsymbol{J}$ RVs computed over different multiple scales. For suitably selected weights, it attains the optimal convergence rate $n^{-1 / 4}$ (Pigorsch [7])

\subsection{Kernel-Based Estimators}

The Kernel-based approach to estimating integrated variance was first used by Zhou [14] to deal with the problem of microstructure noise in high-frequency data. It was later generalized by Hansen and Lunde [15]. Hansen and Lunde [15] studied the properties of Zhou's estimator and showed that, although unbiased under IID noise structure assumption, the estimator is not consistent. As a result, they proposed to estimate IV by:

$$
\mathrm{KRV}_{\mathrm{Z} \& H L}{ }^{\mathrm{m}, \mathrm{H}}=\mathrm{RV}^{\mathrm{m}}+2 \sum_{\mathrm{h}=1}^{\mathrm{H}} \frac{\mathrm{m}}{\mathrm{m}-\mathrm{h}} \gamma_{\mathrm{h}}
$$

with $\gamma_{h}=\frac{m}{m-h} \sum_{i=1}^{m} r_{i}^{(m)} r_{i+h}^{(m)}$

The bias correction factor, $m /(m-h)$, in the above estimator increased the variance of the estimator, so Hansen and Lunde [15] replaced it by the Bartlett kernel and defined the new estimator as:

$$
\mathrm{KRV}^{\mathrm{HL}, \text { Bartlett }}=\mathrm{RV}^{(\mathrm{m})}+2 \sum_{\mathrm{h}=1}^{\mathrm{H}}\left(1-\frac{\mathrm{h}}{\mathrm{H}+1}\right) \hat{\gamma}_{\mathrm{h}}
$$


where $H=\left\lceil\left(\frac{4 m_{t}}{100}\right)^{2 / 9}\right\rceil$ and $\hat{\gamma}_{h}$ is defined as earlier. Nevertheless, their estimator too was inconsistent.

A class of consistent kernel based estimators called realized kernels have been developed by Barndor-Nielsen, Hansen, Lunde, and Shephard [16]. The realized kernels are divided into the flat-top and non-flat-top realized kernels. The flat-top realized kernel of Barndor-Nielsen [16] can be defined as:

$$
K R V_{F T}^{m, H}=R V^{(m)}+\sum_{h=1}^{H} k\left(\frac{h-1}{H}\right)+\left(\hat{\gamma}_{h}+\hat{\gamma}_{-h}\right)
$$

where $k(x)$ for $\in[0 ; 1]$ is a deterministic weight function. If $\mathrm{k}(0)=1, \mathrm{k}(1)=0$ and $\mathrm{H}=\mathrm{cm}^{2 / 3}$, the estimator is asymptotically mixed normal and converges at rate $\mathrm{m}^{1 / 6}$ (Barndorff-Nielsen [17]). The constant $\boldsymbol{c}$ is a function of the kernel and the integrated quarticity and is chosen such that the asymptotic variance of the estimator is minimized. For the flat-top Bartlett kernel where $k(x)=1-x$, the $K R V_{F T}^{m, H}$ estimator has the same asymptotic distribution as the TSRV estimator of Zhang et al.[9] while in the case of a cubic kernel where $k(x)=1-3 x^{2}+2 x^{2}$, its asymptotic distribution is similar to that of the multiple time scale estimator. For smooth kernel functions where $\mathrm{H}=c m^{1 / 2}, k^{\prime}(0)=0$, and $k^{\prime}(1)=0$, the asymptotic distribution of the estimator is mixed normal with the convergence rate of $m^{1 / 4}$.

The non flat-top realized kernels were introduced for practical application. These realised kernels can be defined as:

$$
K R V_{N F T}^{m, H}=R V^{(m)}+\sum_{h=1}^{H} k\left(\frac{h}{H}\right)\left(\gamma_{h}+\gamma_{h-1}\right)
$$

with a convergence rate equal to $m^{1 / 5}$ and a small asymptotic bias:

$$
m^{1 / 5}\left(K R V_{N F T}^{m, H}-I V\right) \stackrel{\mathcal{L} s}{\rightarrow} \mathcal{M} \aleph\left(c^{-2}\left|k^{\prime \prime}(0)\right| \omega^{2}, 4 c k_{0} I Q\right),
$$

where $\mathcal{L} S$ denotes stable convergence and $\mathcal{M} N$ a mixed normal distribution. Though the non flat-top realized kernels are robust to serial dependent noise and to dependence between noise and efficient price, they have a lower convergence rate $\left(\mathrm{m}^{1 / 5}\right)$. The optimal bandwidth, $\boldsymbol{H}$, for the non-fat-top realized kernels is given by:

where $\xi^{2}$ is the signal-to-noise ratio.

$$
H^{*}=c^{*} \xi^{4 / 5} m^{3 / 4}, c^{*}=\left(\frac{k^{\prime \prime}(0)^{2}}{k_{0}}\right)^{1 / 5} \text { and } \xi^{2}=\frac{\omega^{2}}{\sqrt{I Q}},
$$

The optimal value of $\mathrm{H}$ is larger if the variance of the microstructure noise is large in comparison to the integrated quarticity.

A Parzen kernel that is smooth and always produces non-negative estimates has also been suggested by Barndor-Nielsen et al. [21]. This kernel is given by: 


$$
k(x)= \begin{cases}1-6 x^{2}+6 x^{3} \text { for } 0 \leq x<1 / 2 \\ 2(1-x)^{3} & \text { for } 1 / 2 \leq x \leq 1 \\ 0 & \text { for } x>1\end{cases}
$$

For the Parzen kernel, $\boldsymbol{c}^{*}=3.5134$

\subsection{Range-Based Estimation}

The range-based estimator of volatility has been developed since the 1950 s. The estimator is based on the extremes from the entire price path and as a result provides more information than returns sampled at fixed time intervals (Christensen and Podolskij [18]; Pigorsch et al. [7]). It has been found that the squared range based on the daily high and low is about five times more efficient than the daily squared return but however less efficient than RV based on a sampling frequency higher than two hours (Pigorsch, et al. [7]).

Christensen et al. [18] have suggested a realized range-based estimator that replaces the squared intraday returns by normalized squared. By decomposing the daily time interval into $n$ non-overlapping intervals of size, $\Delta$, they obtained the realized rangebased estimator as:

$$
R R V_{m}^{\Delta}=\frac{1}{\lambda_{2, m}} \sum_{i=1}^{n} s_{p i \Delta, \Delta}^{2}, m^{\prime}
$$

where $s_{p i \Delta, \Delta^{\prime}} m^{\prime}=\max _{0 \leq s, t \leq m}\left\{p_{(i-1) / n+t / m n}-p_{(i-1) / n+s / m n}\right\}$ denotes the observed range over the $i$ th interval and $\lambda_{r, m}=E\left[s_{w, m}^{r}\right] \cdot \lambda_{r, m}$ is the $r t h$ moment of the range of a standard Brownian motion over a unit interval, with $\mathrm{m}$ observed increments and $s_{w, m}=\max _{0 \leq s, t \leq m}\left\{W_{t / m}-W_{s / m}\right\}$ is the range of a standard Brownian motion.

In the estimator above, it is assume that the (log) price process follows a continuous semimartingale and that $\Delta+1$ equidistant prices are observed discretely over a day. Also because it is assume that the prices are observed discretely, the estimator is biased downwards. The factor, $\lambda_{2, m}$, in the estimator is therefore meant to correct for the downward bias arising from discretely observed data.

The estimator above has certain advantages over the previous return-and range-based methods. " $R R V_{m}^{\Delta}$ inspects all data points (regardless of $\mathrm{m}$ ), whereby we avoid neglecting information about integrated variance. Second, the efficiency of $R R V_{m}^{\Delta}$ is several times that of RV, leading to narrower confidence intervals for integrated variance" [18, p. 329].

The efficiency of the $R R V_{m}^{\Delta}$ estimator depends on the variance factor, $\boldsymbol{\Lambda}$. For $m_{k}=10$ the factor is about 0.7 . For continuously observed prices, the factor is 0.4 such that RRV is five times more efficient than RV. For $m_{k}=1$ the efficiency of RV is obtained [18].

Market microstructure noise corrections of range-based volatility estimators have been proposed by Martens and van Dijk [19] and Christensen, Podolskij and Vetter [20]. Although bias correction is not as straightforward as in the case of using squared returns, Christensen et al. [20] suggest that bias reduction can be achieved by imposing simple 
parametric assumptions on the distribution of the noise process and sampling at a one to two minutes' frequency.

\subsection{Quantile- Based Estimation}

The quantile-based approach dates back to Pearson [21], Mosteller [22], Eisenberger and Posner [23]. These estimators are based on the quantiles of the log returns rather than the returns themselves; that is, they estimate volatility based on the sample return quantiles. These estimators exploit the fundamental relationship between quantiles and the variance of the normal distribution. For example, the $95 \%$ quantile of an iid Gaussian distribution with zero mean and variance $\sigma^{2}$ is $1.645 \sigma$. So to estimate volatility based on the quantiles of the $\log$ returns, we can invert this relationship. (Christensen, Oomen and Podolskij [24]).

Christensen et al (2009) have developed a quantile-based realized variance (QRV) that combines multiple quantiles for each of the $m_{k}$ intraday subintervals. Construction of this estimator involves splitting the sample into $\boldsymbol{K}$ non-overlapping blocks with $m_{k}$ returns and then the construction of the estimator using the sample quantiles of each of the $\boldsymbol{K}$ subsamples. The QRV of Christensen et al [24] is defined as:

where

$$
Q R V^{\left(m_{k}, K, \bar{\lambda}\right)}=\frac{1}{K} \sum_{i=1}^{p} \alpha_{i} \sum_{j=0}^{k} \frac{q_{j}^{\left(m_{k}, \lambda_{i}\right)}}{v_{1}^{\left(m_{K}, \lambda_{i}\right)}} \quad \text { for } \lambda_{i} \in(1 / 2,1),
$$

- $\alpha=\left(\alpha_{1}, \ldots \alpha_{p}\right)^{\top}$ is a non-negative vector of quantile weights, with the absolute values summing to unity;

- $\quad q_{j}^{m_{k}, \lambda_{i}}=g_{\lambda_{i} m_{K}}^{2}\left(\sqrt{m_{K}} K r_{\left[(j-1) m_{K}+1: j m_{K}\right]}\right) g_{m_{K}-\lambda_{i} m_{K}+1\left(\sqrt{m_{K}} K r_{\left[(j-1) m_{K}+1: j m_{K}\right]}^{2}\right)}$ is the realized squared symmetric $\lambda_{i}-$ quantile of the (scaled) subsample $\mathrm{j}$;

- $\bar{\lambda}=\left(\lambda_{1} \ldots \lambda_{p}\right)^{\top}$ is a vector of $\mathrm{p}$ return quantiles;

- $g l(x)=x_{l}$ is a function that extracts the $l$ th order statistic from a vector $\mathrm{x}$ and

- $v_{1}^{\left(m_{k}, \lambda_{i}\right)}$ is the scaling factor given by:

$v_{r}^{\left(m_{K}, \lambda\right)}=\mathrm{E}\left[\left(\left|U_{(\lambda m k)}\right|^{2}+\left|U_{\left(m_{k}-\lambda m_{k}+1\right)}\right|^{2}\right)^{r}\right]$ with $U_{\left(\lambda m_{k}\right)}$ denoting the $\left(\lambda_{m_{k}}\right)$ th order statistic of an independent standard normal sample $\left\{U_{i}\right\}_{i=1}^{m k}$ (Pigorsh et al [7]).

Christensen et al (24) showed that the above estimator is consistent and robust to jump as $K \rightarrow \infty$. They noted that "as the number of blocks increases, they cover an increasingly short interval so that in the limit and under weak assumptions on the price process, each block contains at most one jump and volatility within the block is locally constant" (Christensen et al, [24], p 75). The term $q_{j}^{\left(m_{k}, \lambda_{i}\right)} / v_{1}^{\left(m_{K}, \lambda_{i}\right)}$ provides an estimator of the (scaled) return variation over the $j$ th block and the sum across all blocks therefore yields a consistent estimator of the IV. [24, p. 75]

The asymptotic distribution of the QRV is given in Pigorsh et al [7] as 
where

$$
\frac{\sqrt{m}\left(Q R V^{\left(m_{k}, K, \bar{\lambda}\right)}-I V\right)}{\sqrt{\theta^{\left(m_{k} \bar{\lambda} \alpha\right.} I Q}} \stackrel{\mathcal{L}}{\rightarrow} \mathcal{N}(0,1)
$$

$$
\begin{aligned}
& \theta^{\left(m_{k} \bar{\lambda} \alpha\right)}=\alpha^{\top} \Theta^{\left(m_{k}, \bar{\lambda}\right)} \alpha \text { and the ijth element of the p x p matrix } \Theta^{\left(m_{k} \bar{\lambda}\right)} \text { is given by } \\
& \Theta_{i, j}{ }^{\left(m_{k}, \bar{\lambda}\right)}=m_{K} \frac{v_{1}^{\left(m_{k}, \lambda_{i} \lambda_{j}\right)}-v_{1}^{\left(m_{k}, \lambda_{i}\right)} v_{1}^{\left(m_{k}, \lambda_{j}\right)}}{v_{1}^{\left(m_{k}, \lambda_{i}\right)} v_{1}^{\left(m_{k}, \lambda_{j}\right)}} \text { with } \\
& v_{1}^{\left(m_{K}, \lambda_{i} \lambda_{j}\right)}=E\left[\left(\left|U_{\left(\lambda_{i} m_{k}\right)}\right|^{2}+\left|U_{\left(m_{k}-\lambda_{m k}+1\right)}\right|^{2}\right)^{2}\left(\left|U_{\left(\lambda_{j} m_{k}\right)}\right|^{2}+\left|U_{\left(m_{k}-\lambda_{j m k}+1\right)}\right|^{2}\right)^{2}\right]
\end{aligned}
$$

For fixed $m_{K}$ and in the absence of noise, Christensen et al [28] showed that QRV estimator converges to the IV at rate $m^{-1 / 2}$ while with microstructure noise, the (modified) estimator converges at rate $m^{-1 / 4}$ Christensen et al.[24] further showed that the QRV estimator can be constructed based on overlapping blocks. The authors proved that such a subsampled version of the estimator further improves its efficiency.

Implementation of the QRV requires choices to be made with regard to the number of blocks $\boldsymbol{K}$, or block length, $\boldsymbol{m}_{\boldsymbol{K}}$, the quantiles $\lambda$, and the quantile weights $\boldsymbol{\alpha}$. For a fixed set of quantiles and block size, Christensen et al. [24] showed that the optimal quantile weights to minimize the asymptotic variance of QRV is given by:

$$
\alpha^{*}=\frac{\left(\Theta^{m, \bar{\lambda}}\right)^{-1} l}{\iota^{\top}\left(\Theta^{m, \bar{\lambda}}\right)^{-1} \iota}
$$

where $\boldsymbol{\iota}$ is a $\boldsymbol{p} \boldsymbol{x} \mathbf{1}$ vector of ones. The selection of quantiles $\lambda$ can be based on efficiency considerations. Christensen et al [24] observed that quantiles near the mode of the distribution are not instructive about the spread of the process while those in the extreme tail of the distribution are erratic. They recommend that the optimal choice of quantile should balance the above trade-off in order to obtain much information about the variance of the distribution. As a result, they identified the optimal quantile as those lying in the region $0.90-0.95$. The authors further noted that quantiles outside $0.90-0.95$ can be used to study the covariance structure of the order statistics when $\boldsymbol{p}>1$. Concerning the block size, Christensen et al. [24] noted that small block size provide modest efficiency gains because they achieve better locality of volatility. However, the gain is insignificant when multiple quantiles are employed.

Christensen et al [24] have also introduced a QRV that is consistent and asymptotically efficient in the presence of microstructure noise. This estimator is defined in Pigorsh et al [7] as:

with

$$
Q R V_{\bar{y}}^{\left(L, m_{k} K \bar{\lambda}\right)}=\frac{1}{c \psi_{2}\left(m-m_{K}(L-1)+1\right)} \sum_{i=1}^{p} \alpha_{i} \sum_{j=0}^{m_{K}(K-L+1)} \frac{q_{\bar{y} ; j}^{\left(m_{K}, \lambda_{i}\right)}}{v_{1}^{\left(m_{K}, \lambda_{i}\right)}}
$$

and

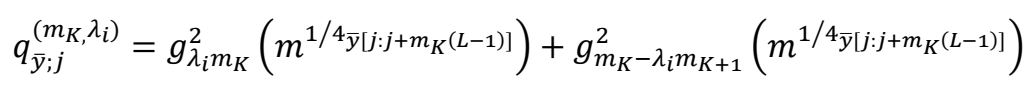




$$
\bar{y}_{j} \text {, the weighted average of the observed returns }=\sum_{i=1}^{L-1} h\left(\frac{i}{L}\right) r_{j+1}^{(m)}
$$

with $L=c \sqrt{m}+o\left(m^{1 / 4}\right)$ for some constant $\boldsymbol{c}$ and weight function $\boldsymbol{h}$ on [0;1].

After carrying out a bias-correction in the above estimator, we get the iid noiserobust estimator:

where $\psi_{1}, \psi_{2}$ are computed by

$$
Q R V_{\text {iid }}^{L, m_{K}, K, \bar{\lambda}}=Q R V_{\bar{y}}^{\left(L, m_{k} K \bar{\lambda}\right)}-\frac{\psi_{1}}{c^{2} \psi_{2}} \omega^{2}
$$

$$
\psi_{2}=L \sum_{j=1}^{L}\left(h\left(\frac{j}{L}\right)-h\left(\frac{j-1}{L}\right)\right)^{2}
$$

and

$$
\psi_{1}=\frac{1}{L} \sum_{j=1}^{L-1} h^{2}\left(\frac{j}{L}\right)
$$

The estimator above converges at rate $m^{-1 / 4}$. Though the asymptotic variance of the modified QRV has no explicit expression in terms of IQ, it can be estimated based on the estimates of the $\boldsymbol{q}_{\mathbf{1}}, \boldsymbol{\psi}_{\mathbf{2}}, \boldsymbol{v}_{\mathbf{1}}$ terms.

Christensen et al. [24] show that for the weight function $h(x)=\min (x, 1-x)$ and in a constant volatility setting, the estimator achieves a lower bound of $8.5 \sigma^{3} \omega$ which is close to the theoretical bound of the variance of the realized kernel approach. The behaviour of the noise robust estimator depends on the choice of $\mathrm{L}$ and there is a trades-off between the noise reduction and the efficiency loss in making this choice due to preaveraging. In practice, the estimated signal-to-noise ratio can be used to select $\boldsymbol{L}$ based on the mean-square error criterion (Pigorsh et al [7]).

\subsection{Duration-Based Estimation}

The duration-based approach focuses on the time it takes the price process to travel between fixed price levels. Although it was Cho and Frees [25] who first investigated this approach for the constant volatility case, a more comprehensive treatment of it was done by Andersen, Dobrev and Schaumburg [26]. Andersen et al [26] investigated the duration-based approach in the case of constant volatility and for stochastic volatility evolving without drift by considering three different ways in which we can measure the time it takes a Brownian motion to travel a given distance, $h$. The three measures of duration (also called "passage time") are:

first exit time : $\tau_{h}=\inf \left\{t>0|| B_{t} \mid>h\right\}$

first range time : $\tau_{h}=\inf \left\{t>0 \mid \max _{0 \leq s \leq t} B_{s}-\min _{0 \leq s \leq t} B_{s}>h\right\}$

first heating time : $\tau_{h}=\inf \left\{t>0 \mid B_{t}=h\right\}$

Using moment generating functions, Andersen et al [26] derived the moments of the above passage times for the constant volatility case as: 


$$
E\left[\tau_{h}\right]=\left\{\begin{array}{c}
\frac{h^{2}}{h^{2}} \text { (first exit time) } \\
\frac{1}{2} \frac{h^{2}}{\sigma^{2}} \text { (first range time) } \\
\infty \quad \text { (first hitting time) }
\end{array}\right.
$$

Based on the above moment conditions, the authors obtained a method of moments estimator of volatility using an observed sample of passage times with fixed $h$ (except in the case of the first hitting time which does not have a first moment). A problem in using the above moment conditions to estimate $\boldsymbol{\sigma}^{2}$ is that they can suffer from quite severe small sample biases induced by Jensen's inequality. This is because the expected passage time is inversely proportional to the instantaneous variance" [26, p. 12]. Andersen showed that for a given sample of passage times of size $\mathrm{N}$

$$
E\left[\frac{h^{2}}{\frac{1}{N} \sum_{i=0}^{N-1} \tau_{i, h}}\right]=\mu_{1}^{(N)} \sigma^{2} \text { where } \mu_{1}^{(N)}=\left\{\begin{array}{c}
1+\frac{2}{3 N}+O\left(\frac{1}{N^{2}}\right) \quad \text { (first exit time) } \\
1+\frac{1}{3 N}+O\left(\frac{1}{N^{2}}\right) \quad(\text { first range time) } \\
\left.\left.\frac{1}{N} \quad \text { (first hitting time }\right)\right)
\end{array}\right.
$$

In view of the above, Andersen proposed a small sample unbiased estimator based on the first moment of the reciprocal passage times:

$$
E\left[\begin{array}{l}
h^{2} \\
\tau^{h}
\end{array}\right]=\mu_{1} \sigma^{2}=\left\{\begin{array}{c}
\left.2 C \sigma^{2} \text { (first exit time }\right) \\
\left.(4 \log 2) \sigma^{2} \text { (first range time }\right) \\
\sigma^{2} \quad(\text { first hitting time })
\end{array}\right.
$$

where $\mathrm{C} \approx 0.916$ is the Catalan constant. The moments of the reciprocal passage times also allow us to define a local volatility estimator for a single passage time using a single observation transition

$$
\hat{\sigma}_{h}^{2}=\frac{1}{\mu_{1}} \frac{h^{2}}{\tau_{h}}
$$

such that integrated variance can also be estimated in the case of stochastic volatility by applying the Riemann sum.(Pigorsh et al. [7, p. 13]).

In the stochastic volatility case, Andersen et al. [26] showed that the local volatility estimator can be constructed using either the previous passage time or the next passage time or both. Thus, the authors identified two independent estimators of local volatility at time $\boldsymbol{t}_{\boldsymbol{i}}$ as follows:

$$
\begin{array}{ll}
\hat{\hat{\sigma}}_{h}^{2}\left(t_{i}\right)=\frac{1}{2 \mu_{1}}\left[\frac{h^{2}}{\tau_{h}^{+}\left(t_{i}\right)}+\frac{h^{2}}{\tau_{h}^{-}\left(t_{i}\right)}\right] & \text { bi-directional } \\
\hat{\sigma}_{h}^{2}\left(t_{i}\right)=\frac{1}{\mu_{1}} \frac{h^{2}}{\tau_{h}^{+}\left(t_{i}\right)} \text { or } \frac{1}{\mu_{1}} \frac{h^{2}}{\tau_{h}^{-}\left(t_{i}\right)} \quad \text { uni-directional }
\end{array}
$$

However, they noted that "in practice, we do not use the bi-directional estimators due to censoring issues. Instead we use the uni-directional estimator based on $\tau_{h}^{+}\left(t_{i}\right)$ for grid 
points $t_{i}$ that fall in the first half of the trading day and $\tau_{h}^{-}\left(t_{i}\right)$ for grid points falling in the second half of the trading day". (Andersen, et al, 2009, p 19). This suggestion "is based on their simulation results for exit and range passage times showing that left and right censoring can be ignored, if the difference in time to the market opening and closing is 2 to 3 times longer than the expected passage times." [7]. The censoring problem mentioned above is induced by market closures "In particular, the expected next passage time is affected by the time left until the market closes, (right censoring), while the expected previous passage time is limited by the time the market opened, (left censoring)." [7, p. 14].

By using a sequence of local variance estimates, $\left\{\hat{\sigma}_{h}^{2}\left(t_{i}\right)\right\} i=1, \ldots N$ based on passage time durations, Andersen et al. [30] constructed the estimate of IV on [0,1] as:

$$
\widehat{D R V}_{N, h}=\sum_{i=1}^{N-1} \hat{\sigma}_{h}^{2}\left(t_{i}\right) \Delta_{i}
$$

with $\Delta_{i}=\frac{1}{N}$ denoting the times between the intraday observations in the case of an equispaced time grid.

The asymptotic distribution of the DRV is given as:

$$
\sqrt{N}\left(\widehat{D R V}_{N, h}-I V\right) \sim \operatorname{Mixed} \text { Normal }\left(0, v \int_{0}^{1} \sigma_{u}^{4} d u\right)
$$

where $v$ is a constant that is specific to the type of passage time used in the estimation and that is independent of the choice of $\boldsymbol{h}$. (Pigorsh et al. [7]). The integrated quarticity of the estimator can be consistently estimated as:

$$
\widehat{D R V}_{N, h}=\sum_{i=1}^{N}\left(\widehat{\Delta}_{i}\right)^{4} \Delta_{i}
$$

to enable the calculation of confidence bands for IV (Andersen et al. [26]).

The asymptotic efficiency of the DRV is higher than that of the return-based estimators especially if the dataset allows the usage of bi-directional passage times through non-interrupted trading. However "in practice, the observation record is discrete and we only observe the value of the process at the $\boldsymbol{N}$ grid points, thus rendering the convergence rate $N^{-1 / 2}$ of the estimator $\widehat{D R V}_{N, h}$ infeasible" [26, p. 20]. Andersen therefore suggest to sample sparsely in order to avoid this potentially more pronounced discreetness effect. DRV based on first range time and on first exit time may be biased, because the observed times may not be the same as the true ones.

The results of the simulation study of Andersen indicated that the DRV estimator is sufficiently robust to independent noise with moderate levels of noise-to-signal ratio even in the case of first range and first exit times. Higher threshold values of $\boldsymbol{r}$ make the estimator more robust to noise. 


\subsection{Jump-Robust Realized Measures}

Suppose the $\log$ price of a financial asset, $\boldsymbol{p}_{\boldsymbol{t}}$, follows the continuous-time semimartingale jump diffusion process:

$$
p_{t}=\int_{0}^{t} \mu(s) d s+\int_{0}^{t} \sigma(s) d W(s)+\sum_{j=1}^{N(t)} k\left(s_{j}\right)
$$

where the mean process $\mu(t)$ is continuous and of finite variation, $\sigma(s)>0$ denotes the càdlàg instantaneous volatility, $\mathrm{W}(\mathrm{t})$ is a standard Brownian motion and the $\mathrm{N}(\mathrm{t})$ process counts the number of jumps occurring with possibly time-varying intensity $\lambda(t)$ and jump size $k\left(s_{j}\right)$. Given the above, the quadratic variation of $\boldsymbol{p}_{\boldsymbol{t}}$, can be decomposed into a component due to continuous variation (integrated variance, or IV) and a component due to jumps (denoted JV):

$$
Q V_{t}=\operatorname{plim}_{n \rightarrow \infty} \sum_{j=1}^{n} r_{t+1 / j}^{2}=\underbrace{\int_{0}^{t} \sigma^{2}(s) d s}_{I V_{t}}+\underbrace{\sum_{j=1}^{N(t)} k^{2}\left(s_{j}\right)}_{J V_{t}}
$$

A natural estimator of the realized quadratic variation is realized variance or realized volatility. From the theory of quadratic variation, it follows that the basic RV estimator converges uniformly in probability to the quadratic variation as the sampling frequency of the underlying returns approaches infinity:

$$
R V_{t} \stackrel{p}{\rightarrow} \underbrace{\int_{t-1}^{t} \sigma^{2}(s) d s}_{I V_{t+1}}+\underbrace{\sum_{j=N(t-1)+1}^{N(t)} k^{2}\left(s_{j}\right)}_{J V_{t+1}}
$$

(Bollerslev, Kretschmer, Pigorsch \&Tauchen [27]).

However, the need to isolate and estimate the integrated variance in the presence of possible jumps has led to the development of various estimators which attempt to exclude jump variation. The most common realized measures that estimate only the integrated variance, i.e. 'jump-robust' realized measures include:

1) the bi-power variation (BPV) which is defined as :

$$
B P V^{(m)}=\frac{\pi}{2} \sum_{j=2}^{m}\left|r_{t, j}\right|\left|r_{t, j-1}\right|
$$

"For increasingly finely sampled returns, the BPV measure becomes immune to jumps and consistently (for increasing values of $\boldsymbol{m}$ ) estimates the integrated variance" [27, p.3]. Huang and Tauchen [28] have proposed the relative jump statistic, $R J_{t}=$ $\left(R V_{t}-B P V_{t}\right) R V_{t}^{-1}$ or its $\log$ arithmic version $J_{t}=\log R V_{t}-\log B P V_{t}$ as a more robust measure. A problem with the BPV estimator is that it is biased in finite samples. "This bias arises from the fact that in finite samples the diffusive return does not equal zero 
and thus the jump return is not completely cancelled out. This drives up the estimated value of IV and creates an upward bias" (Carlston [29, p. 27])

2) the MedRV and MinRV measures. They are the square of the minimum of two adjacent absolute returns or the median of three adjacent absolute returns. The MedRV and MinRV measures have higher levels of robustness in the presence of jumps and microstructure noise compared to the BPV. The MedRV was introduced to deal with outliers or wrongly recorded observations" [11, p. 296].

3 ) the threshold realized variance developed in Mancini [30, 31]. This estimator is the sum of squared returns, which are less than a certain threshold. The construction of this estimator relies on filtering out returns that exceed a certain threshold thus enabling the elimination of large returns that are the result of jumps in the price process and the inclusion of only diffusive returns in the estimator. The appropriate choice of threshold is a major difficulty associated with this estimator.

4) the quantile-based realized variance (QRV) as discussed earlier. In finite samples, the QRV estimator performs better than the BPV when there are jumps in the price process. It is also consistent and efficient when the observed log price is contaminated by market microstructure noise.

\section{Empirical Evaluation of the Performance of Realized Volatility Estimators}

Patton [32] studied the estimation accuracy of the standard RV estimators by combining:

a) two different price series: trade prices and mid-quote prices,

b) two different sampling schemes: calendar-time sampling and tick-time sampling and

c) thirteen different sampling frequencies :1, 2, 5, 15, 30 seconds, 1, 2, 5, 15, 30 minutes, 1, 2 hours and the open-close return.

The combination of two price series, two sampling schemes and 13 sampling frequencies resulted in 48 possible RV estimators. The analysis was based on IBM stock returns data over the period January 1996-June 2007. Patton's study revealed that for both sampling schemes, standard RV estimators based on trade prices were positively biased at very high sampling frequencies while those based on quote prices showed a negative bias at the same frequencies. The result of the study also indicated that there was little or no bias at all at lower sampling frequencies (5 minutes and lower) under the different price series and sampling schemes.

Patton [32] also analysed the performance of the $48 \mathrm{RV}$ estimators identified above relative to the 5-min calendar-time RV on trade prices. He employed the QLIKE distance measure to compare the average distance of the $48 \mathrm{RV}$ estimators and 5-min calendar-time RV on trade prices from the latent quadratic variation of the IBM price process. The results of his analysis revealed that RV estimators computed on returns sampled at lower frequencies (30-min or lower) performed poorly than those computed on higher-frequency data. In particular, the higher frequency based RV estimators had shorter estimated average distances from the true QV under the random walk (RW) approximation for the dynamics in QV. Patton found the best-performing estimator to be the 1-min tick time RV computed on trade prices. 
Patton (2011) further examined the performance of the $48 \mathrm{RV}$ estimators relative to a "benchmark" RV estimator using the stepwise multiple testing approach of Romano and Wolf [33]. The above method helps to pinpoint estimators that are significantly better, or worse than a given benchmark estimator [29]. Using the squared open-to-close return computed using calendar-time trade price as a benchmark RV estimator, Patton discovered that with the exception of the squared open-to-close quote-price return, all the other estimators were significantly better than the benchmark at the 0.05 level. However, he found that the open-to-close quote-price RV estimator was not significantly different from the open-to-close trade-price RV under both the RW and autoregressive (AR) approximations for the dynamics in QV.

Also using a 5-min calendar-time trade price RV estimator as a benchmark RV estimator, Patton [32] established that higher-frequency RV estimators were significantly better than the benchmark RV. In particular, RV estimators computed at 15-s to 2-min sampling frequencies were significantly better than 5-min RV under the RW approximation. The result also showed that estimators computed at ultra-high sampling frequencies (5-s to 1-s) were not significantly different from the benchmark estimator while those computed at 15-min or lower sampling frequencies were found to be significantly worse than the 5-min RV estimator.

Under the AR approximation, majority of the estimators were not significantly different from the 5-min calendar-time trade price RV estimator. In particular, all the estimators computed on trade prices sampled in calendar and tick time were no different from the benchmark RV estimator. It was only RV computed on quote prices sampled in calendar and tick at frequencies ranging from 5-min to 2-s and 1-min to 5-s respectively that appeared to be significantly worse than the benchmark RV estimator. This points to the fact that the benefits from sampling at frequencies higher than 5-min are offset by the additional estimation error from the AR model.

Patton [32] also compared the performance of the TSRV, MSRV, KRV, RRV and RV computed using calendar-time trade prices sampled at $1 \mathrm{~s}, 5$ min and 1 day against a 5-min calendar-time trade price RV estimator via a bootstrap version of the Diebold and Mariano [34] test. For the TSRV, he used one tick as the highest frequency and the optimal "sparse" sampling frequency identified in Zhang et al. [9]. For MSRV, he used one tick as the highest frequency and adopted the formula of Zhang [13] for the frequencies of the other estimates and the weights used to combine these estimates. For the $\mathrm{KRV}$, he used the modified "Tukey-Hanning 2 " kernel based on 1-min tick-time sampling and adopted the approach in Barndorff-Nielsen et al. [17] to determine the bandwidth. For the RRV he used 5-min blocks with 1-min prices within each block. Under the RW approximation for the dynamics of QV, Patton [32] found that the TSRV, MSRV, KRV and RRV estimators out-performed the simple 5-min RV estimator. In particular, the KRV and RRV estimators appeared to be superior to all the other estimators. However, under the AR approximation, the estimation accuracy of TSRV, MSRV, KRV, and RRV was not better than the simple 5-min RV estimator.

Bandi and Russell [35] and Bandi, Russell and Zhu [36] employed a reduced-form model to study the performance of optimally-sampled realized variances (covariances) and realized variances (covariances) based on ad-hoc sampling intervals in predicting variances (covariances) out-of-sample. They found that optimally-sampled realized variances performed better than realized variances based on ad-hoc sampling interval. 
The MIDAS approach of Ghysels, Santa-Clara and Valkanov (2006.) was adopted in Ghysels and Sinko [37] to assess the performance of realized variance constructed using fixed intervals, realized variance corrected for bias and power variation. Power variation was found to be the best performing estimator.

Large $[38,39]$ analysed the forecasting performance of the alternation estimator and realized variance based on ad-hoc, fixed intervals by employing the HAR-RV model of Corsi (2003). The findings indicate that the alternation estimator is superior to realized variance based on ad-hoc, fixed intervals.

A comparison between the two-scale estimator and realized variance based on different simulation set up for stochastic volatility and microstructure noise has been done by Aït-Sahalia and Mancini [40]. A comparison based on the $R^{2}$ of the Mincer-Zarnowitz (MZ) regression was also done by the authors. The findings showed that the two scale estimator performed better than the realized variance both in terms of mean square error (MSE) and forecasting ability.

Liu, Patton and Sheppard [11] compared various realized variance estimators with the 5-minute RV estimator. They evaluated 400 different estimators based on 11 years of data on 31 different financial assets. Based on the testing approach of Romano and Wolf (2005), they found no evidence that other realized variance estimators performed better than the benchmark 5-minute RV estimator. However using the model confidence set approach, they established that the more sophisticated measures (most notably 1-minute sub-sampled RV, and 1- and 5-second realized kernels and MSRV) outperform it.

Andersen, Bollerslev and Meddahi [41] employed Mincer-Zarnowitz style regression to assess the performance of alternative realized volatility estimates derived from stochastic volatility models . In Andersen et al. (2006), the $R^{2}$ (of the MZ regression) figures reported for the one-step-ahead forecasts, showed that the average estimator, $R_{t}^{\text {average }}$, which averages $R_{t}^{\text {all }}, R_{t}^{\text {sparse }}, R_{t}^{T S}, R_{t}^{\text {Zhou }}$ and $R_{t}^{\text {Kernel }}$ ) dominates uniformly both as the basis for forecasts and as the proxy for the future realized return variation.

Ghysels and Sinko [42] employed the MIDAS regression to conducted empirical study of forecasting with microstructure noise using the 30 Dow Jones stocks. The results of their study revealed that the subsampling and averaging approach represent the class of estimators that performs best in a prediction context.

Other studies also have examined the forecasting performance of the realized volatility estimators from the point of view of economic criteria. Two economic metrics (utility based and profit-based or preference free evaluation) have been applied so far. Bandi and Russell $[43,44]$ assessed the forecasting performance of realized volatility estimators based on the long-run utility that a Mean-variance investor would obtain from using alternative realized volatility forecast in making a portfolio choice. A parallel analysis in the multivariate case has been done by Bandi and Russell [45] and De Pooter, Martens and Van Dijk [46]. A comparison of the forecasting performance of the optimally sampled realized volatility estimators and fixed-interval realized volatility measures using the profit-based evaluation criterion has been done by Bandi and Russell [47]. The results of these studies showed the optimally sampled realized variance estimators to be superior to realized variance based on fixed intervals in forecasting volatility. They also favour the optimized consistent estimators (e.g. two-scale estimator) in out of sample volatility forecasting. 
Bandi, Russell, and Yang [48] used profit or loss that option dealers would obtain from trading on the basis of alternative volatility forecasts to evaluate realized volatility estimators. They adopted a methodology developed in Engle et al. (1990) and as a result operated in a simulated option market. They found the optimized (in a finite sample) flat-top kernel estimators to be the best estimators with regard to average profits from trading and Sharpe ratios. They also found that the near consistent Bartlett kernel estimator, the (bias-corrected and unadjusted) two scale estimator of Zhang et al. (2005) and the flat-top kernel estimators (when the number of subsamples/autocovariances is carefully chosen using finite sample Methods) can perform very well. However, the study confirmed that the optimally sampled realized variance almost always performed better than the 5- and 15-min realized variance.

\section{Conclusion}

At the moment, there is a fairly large number of realized volatility estimators. Each year, new and more complex methods are introduced. This article reviewed both theoretical and empirical literature on the performance of realized measures of quadratic variation/ integrated variance of financial asset returns so as to facilitate their practical application in derivative pricing and hedging, asset allocation and day to day risk management. The results of the review shows that although no single realized measure performs best in all situations and under all circumstances, the more sophisticated realised measures, in particular the TSRV and KRV are superior to the other estimators in terms of their estimation accuracy in the presence of market microstructure noise.

\section{References}

1. Andersen T. G., Bollerslev T., Diebold F. X., Labys P. (2000). Understanding, Optimizing, Using and Forecasting Realized Volatility and Correlation. Great Realizations, Risk, p. 105-108.

2. Aït-Sahalia Y., Yu J. (2009). High Frequency Market Microstructure Noise Estimates and Liquidity Measures. The Annals of Applied Statistics, vol. 3, no. 1, p. 422-457. DOI 10.1214/08-AOAS200

3. Sizova N. (2011). Integrated variance forecasting: Model based vs reduced form. Journal of Econometrics, vol. 162, iss. 2, p. 294-311.

4. Andersen T. G., Bollerslev T., Diebold F. X., Labys P. (2001). The Distribution of Realized Exchange Rate Volatility. Journal of the American Statistical Association, 96: 453, 42-55. DOI 10.1198/016214501750332965

5. Andersen T. G., Bollerslev T., Diebold X. F., Labys P. (2003). Modeling and Forecasting Realized Volatility. Econometrica, vol. 71, no. 2, p. 579-625.

6. Barndorff-Nielsen O. E., Shephard N. (2002). Estimating quadratic variation using realized variance. Journal of Applied Econometrics, vol. 17, iss. 5, p. 457477.

7. Pigorsch C., Pigorsch U., Popov I. (2012) Volatility Estimation Based on HighFrequency Data. In: Duan J. C., Härdle W., Gentle J. (eds) Handbook of Computational Finance. Springer Handbooks of Computational Statistics. Springer, Berlin, Heidelberg. 
8. Barndor-Nielsen and Shephard (2002) Econometric analysis of realized volatility and its use in estimating stochastic volatility models. Journal of the Royal Statistical Society Series B, 2002, vol. 64, iss. 2, p. 253-280.

9. Zhang L., Mykland P. A., Aït-Sahalia Y. (2005). Tale of Two Time Scales. Journal of the American Statistical Association, 100: 472, p. 1394-1411. DOI 10.1198/016214505000000169

10. McAleer M., Medeiros M. C. (2008). Realized Volatility: A Review. Econometric Reviews, 27 (1-3): 10-45.

11. Liu L. Y., Patton A. J., Sheppard K. (2015). Does anything beat 5-minute RV? A comparison of realized measures across multiple asset classes. Journal of Econometrics, vol. 187, iss. 1, p. 293-311.

12. Zhang, Lan \& Mykland, Per A. \& Aït-Sahalia, Yacine, 2011. Edgeworth expansions for realized volatility and related estimators. Journal of Econometrics, Elsevier, vol. 160 (1), p. 190-203.

13. Zhang L. (2006). Efficient estimation of stochastic volatility using noisy observations: a multi-scale approach. Bernoulli, 12 (6), 1019-1043.

14. Zhou Bin. High-Frequency Data and Volatility in Foreign-Exchange Rates. Journal of Business \& Economic Statistics, 1996, vol. 14, iss. 1, p. 45-52.

15. Hansen P. R., Lunde A. (2006). Realized Variance and Market Microstructure Noise. Journal of Business \& Economic Statistics, 24: 2, 127-161. DOI 10.1198/ 073500106000000071

16. Barndor-Nielsen, Hansen, Lunde, and Shephard. Designing Realized Kernels to Measure the ex post Variation of Equity Prices in the Presence of Noise. Econometrica, 2008, vol. 76, iss. 6, p. 1481-1536.

17. Barndorff-Nielsen O. E., Hansen P. R., Lunde A., Shephard N. (2009). Realized kernels in practice: trades and quotes. The Econometrics Journal, vol. 12, iss. 3, p. C1-C32. DOI 10.1111/j.1368-423X.2008.00275.x

18. Christensen K., Podolskij M. (2007). Realized range-based estimation of integrated variance. Journal of Econometrics, vol. 141, iss. 2, p. 323-349.

19. Martens M., Dijk D. (2007). Measuring volatility with the realized range. Journal of Econometrics, vol. 138, iss. 1, p. 181-207.

20. Christensen K., Podolskij M., Vetter M. (2009). Bias-correcting the realized range-based variance in the presence of market microstructure noise. Finance and Stochastics, vol. 13, iss. 2, p. 239-268.

21. Pearson, G. A. Factors controlling the distribution of forest types, Part I. Ecology, vol. 1, 1920, p. 139-154.

22. Mosteller, F. On some useful "inefficient" statistics. Annals of Mathematical Statistics, 17, 1946, p. 377-408.

23. Isidore Eisenberger and Edward C. Posner Systematic Statistics Used for Data Compression in Space Telemetry. Journal of the American Statistical Association, vol. 60, no. 309 (Mar., 1965), p. 97-133.

24. Christensen, Kim and Oomen, Roel C. A. and Podolskij Mark. Realised Quantile-Based Estimation of the Integrated Variance (September 1, 2009). Journal of Econometrics, vol. 159, no. 1, p. 74-98. 2010. Available at SSRN: https:// ssrn.com/abstract=1085553 
25. D. Cho, R. Frees. Estimating the volatility of discrete stock prices. Journal of Finance, 43, 1988, p. 451-466.

26. Andersen T. G., Dobrev, D., Schaumburg E. (2009). Duration-Based Volatility Estimation. http://hdl.handle.net/10086/17081

27. Bollerslev T., Kretschmer U., Pigorsch C., Tauchen G. (2009). A discrete-time model for daily S \& P500 returns and realized variations: Jumps and leverage effects. Journal of Econometrics, vol. 150, iss. 2, p. 151-166.

28. Huang X., Tauchen G. (2005). The Relative Contribution of Jumps to Total Price Variance. Journal of Financial Econometrics, vol. 3, iss. 4, p. 456-499. DOI 10.1093/ijfinec/nbi025

29. Carlston B. (2017). Data-Based Ranking of Integrated Variance Estimators Across Size Deciles. Journal of Economic Theory and Econometrics, vol. 28, no. 1, p. 2148.

30. Mancini, C. Disentangling the jumps of the diffusion in a geometric jumping Brownian motion. G. dell. Ital. degli Attuari, 64, 19-47, 2001.

31. Mancini, C. Non-parametric threshold estimation for models with stochastic diffusion coefficient and jumps. Scand. J. Stat., 36 (2), 270-296, 2009.

32. Patton A. J. (2011). Data-based ranking of realised volatility estimators. Journal of Econometrics, vol. 161, iss. 2, p. 284-303.

33. Romano, J. P., Wolf, M. Stepwise multiple testing as formalized data snooping. Econometrica, 73 (4), 1237-1282, 2005.

34. Diebold, F. X., Mariano, R. S. Comparing predictive accuracy. Journal of Business and Economic Statistics, 13, 253-263, 1995.

35. Bandi, F. M., Russell, J. R. 2006. Separating microstructure noise from volatility. Journal of Financial Economics, 79, 655-692.

36. Bandi, F. M., Russell, J. R., Zhu, Y. (2008). Using high-frequency data in dynamic portfolio choice. Econometric Reviews, 27, 163-198.

37. Ghysels, E., Sinko, A. 2006. Comment on Hansen and Lunde. Journal of Business and Economic Statistics, 24, 192-194.

38. Large J. 2006. Estimating quadratic variation when quoted prices change by constant increments. Working Paper.

39. Large J. (2011). Estimating quadratic variation when quoted prices change by a constant increment. Journal of Econometrics, vol. 160, iss. 1, January 2011, p. 2 11.

40. Aït-Sahalia, Y., Mancini, L. (2008). Out-of-sample forecasts of quadratic variation. Journal of Econometrics, 147 (1), 17-33.

41. Andersen, T. G., Bollerslev, T., Meddahi, N. 2006. Realized volatility forecasting and market microstructure noise. Working Paper.

42. Ghysels, E., Sinko, A. 2006. Volatility forecasting and microstructure noise. Working Paper.

43. Bandi, F. M., Russell, J. R. 2005. Market microstructure noise, integrated variance estimators, and the accuracy of asymptotic approximations. Working Paper.

44. Bandi, F. M., Russell, J. R. 2006. Separating microstructure noise from volatility. Journal of Financial Economics, 79, 655-692.

45. Bandi, F. M., Russell, J. R. (2008). Volatility. In: Birge, J., Linetski, V. (Eds.), Handbook of Financial Engineering. Elsevier, North Holland. 
46. De Pooter, M., Martens, M., Van Dijk, D. (2008). Predicting the daily covariance matrix for S\&P100 stocks using intraday data: But which frequency to use? Econometric Reviews, 27, 199-229.

47. Bandi, F. M., Russell, J. R. (2008). Microstructure noise, realized variance, and optimal sampling. Review of Economic Studies, 75, 339-369.

48. Bandi F. M., Jeffrey R. Russell J. R. and Yang C. (2008). Realized volatility forecasting and option pricing. Journal of Econometrics, 147, 34-46.

Материал поступил в редколлегию 10.02.2020

Принят к печати 29.06.2020

The article was submitted 10.02.2020

Accepted for publication 29.06.2020

\section{Сведения об авторах}

Гайомей Джон, аспирант Новосибирского государственного университета (Новосибирск, Россия)

gayomey@hotmail.com

Костин Андрей Владимирович, кандидат экономических наук, старший научный сотрудник, Институт экономики и организации промышленного производства СО РАН (Новосибирск, Россия), доцент, Новосибирский государственный университет (Новосибирск, Россия)

a.kostin@nsu.ru

SPIN-код 8764-9125

ORCID 0000-0002-9337-3978

AuthorID 696438

Scopus authorID 57206934882

\section{Information about the Authors}

John Gayomey, Graduate Student of Novosibirsk State University (Novosibirsk, Russian Federation)

gayomey@ hotmail.com

Andrei V. Kostin, Candidate of Economics, Senior Researcher, Institute of Economics and Industrial Engineering of the Siberian Branch of the Russian Academy of Sciences (Novosibirsk, Russian Federation), Associate Professor, Novosibirsk State University (Novosibirsk, Russian Federation)

a.kostin@nsu.ru

SPIN-код 8764-9125

ORCID 0000-0002-9337-3978

AuthorID 696438

Scopus authorID 57206934882 